\section{P2-268 RISK OF CARDIOVASCULAR DISEASE IN DIABETICS WITH SYMPTOMS OF CARDIAC ISCHAEMIA IN WESTERN REGION OF NEPAL: A HOSPITAL BASED STUDY}

doi:10.1136/jech.2011.142976k.1

${ }^{1} B$ Sathian, ${ }^{*}$ 'A Kumari, ${ }^{2} J$ Sreedharan, ${ }^{1}$ A Mittal. ${ }^{1}$ Manipal College of Medical Sciences, Pokhara, Nepal; ' Gulf Medical University, Ajman, United Arab Emirates

Background The cardiovascular (CV) risk in patients with diabetes is estimated to be two or three times higher than in the non-diabetic population. The prognosis of patients with diabetes following myocardial infarction (MI) remains poorer in comparison with nondiabetics.

Objective To find out the relationship between Diabetes and MI in patients with symptoms of cardiac ischaemia.

Materials and Methods It was a retrospective study carried out from the data maintained in the Department of Biochemistry of the Manipal Teaching Hospital, Pokhara, Nepal between 1 January 2008 and 1 January 2009. Data of 422 patients with symptoms of cardiac ischaemia who underwent both Troponin and lipid profiles tests were collected. Descriptive statistics and testing of hypothesis were used for the analysis using SPSS V. 16 software.

Result Of the 422 subjects, $10.4 \%$ were Troponin positive, $62.1 \%$ Diabetic, $51.4 \%$ greater than 60 year old. Average age of Troponin positive was 67.2 and negative was 58.3 years. A relationship was found to exist between Diabetes and MI $(O R=15, p<0.0001)$, especially in age group greater than 60 year old $(O R=10, p<0.0001)$. Conclusion CV risk should be assessed in all patients with diabetes. Those with a high risk should be treated intensively, and this needs to be clearly explained by their doctors in order to improve compliance.

\section{P2-269 REGIONAL DIFFERENCES IN THE PREVALENCE OF TYPE 2 DIABETES MELLITUS: RESULTS FROM FIVE POPULATION- BASED COHORT STUDIES IN GERMANY (DIAB-CORE CONSORTIUM)}

doi:10.1136/jech.2011.142976k.2

${ }^{1} \mathrm{~S}$ Schipf, ${ }^{*}{ }^{1} \mathrm{~A}$ Werner, ${ }^{2} \mathrm{R}$ Holle, ${ }^{2} \mathrm{M}$ Schunk, ${ }^{3} \mathrm{C}$ Meisinger, ${ }^{3} \mathrm{~B}$ Thorand, ${ }^{4} \mathrm{~K}$ Berger, ${ }^{4} \mathrm{G}$ Müller, ${ }^{5} \mathrm{~S}$ Moebus, ${ }^{6} \mathrm{~A}$ Kluttig, ${ }^{7} \mathrm{~K}$ H Greiser, ${ }^{8} \mathrm{H}$ Neuhauser, ${ }^{8} \mathrm{U}$ Ellert, ${ }^{9} \mathrm{~A}$ Icks, ${ }^{9}$ T Tamayo, ${ }^{9} \mathrm{~W}$ Rathmann, ${ }^{1} \mathrm{H}$ Völzke. ${ }^{1}$ Institute for Community Medicine, University of Greifswald, Greifswald, Germany; ${ }^{2}$ Institute of Health Economics and Health Care Management, German Research Center for Environmental Health, Helmholtz Zentrum München, Neuherberg, Germany; ${ }^{3}$ Institute of Epidemiology II, German Research Center for Environmental Health, Helmholtz Zentrum München, Neuherberg, Germany; ${ }^{4}$ Institute of Epidemiology and Social Medicine, University of Münster, Münster, Germany; ${ }^{5}$ Institute for Medical Informatics, Biometry and Epidemiology, University Hospital of Essen, University Duisburg-Essen, Essen, Germany; ${ }^{6}$ Institute of Medical Epidemiology, Biostatistics and Informatics, Martin-Luther-University Halle-Wittenberg, Halle (Saale), Germany; ' Division of Cancer Epidemiology, German Cancer Research Center (DKFZ), Heidelberg, Germany; ${ }^{8}$ Department of Epidemiology, Robert Koch Institute, Berlin, Germany; ${ }^{9}$ Institute of Biometrics and Epidemiology, German Diabetes Center, Leibniz Center for Diabetes Research at Heinrich-Heine-University, Düsseldorf, Germany

Introduction In Germany, regional data on the prevalence of type 2 diabetes mellitus (T2DM) are lacking for use in healthcare planning. We analysed regional differences in the prevalence of T2DM and treatment with antidiabetic agents.

Methods Data from five regional population-based studies and the German National Health Interview and Examination Survey (GNHIES98) conducted between 1997 and 2006 were analysed. Estimates of self-reported diabetes, treatment, and onset of diabetes were compared. T2DM prevalence (95\% CI) for the five regional studies was directly standardised to the German adult population (31 December 2007).
Results From 11688 participants aged 45-74 years, 1008 had prevalent T2DM, corresponding to a prevalence of $8.6 \%(8.1 \%-9.1 \%)$. For the GNHIES98 a prevalence of $8.2 \%(7.3 \%-9.2 \%)$ was estimated. Prevalence was higher in men $(9.7 \% ; 8.9 \%-10.4 \%)$ than in women $(7.6 \% ; 6.9 \%-8.3 \%)$. The age-standardised regional prevalence was highest in the East with $12.0 \%(10.3 \%-13.7 \%)$ and lowest in the South with $5.8 \%(4.9 \%-6.7 \%)$. The mean age of onset of T2DM was lower in Northeast (53+9SD) than in West-Germany $(57+10 S D)$. Treatment with oral antidiabetic agents was more frequently reported in the South (56.9\%) than in the Northeast $(46.0 \%)$, whereas treatment with insulin alone was more frequently reported in the Northeast (21.6\%) than in the South (16.4\%).

Conclusion The prevalence of T2DM showed a Northeast and Southwest gradient within Germany with the highest standardised prevalence in the East, which is in accordance with regional differences in the distribution of risk factors for T2DM. Furthermore, the treatment with antidiabetic agents showed regional differences.

\section{P2-270 HABIT OF REMOVING VISIBLE FAT FROM MEATS BY ADULTS AND ELDERLY: A POPULATION-BASED STUDY IN SOUTHERN BRAZIL}

doi:10.1136/jech.2011.142976k.3

1,2B Schneider, ${ }^{*}$ 1,2S Silva, 1,2M C Assunção. ' Universidade Federal de Pelotas, Pelotas, Rio Grande do Sul, Brazil, ${ }^{2}$ Programa de Pós Graduação em Epidemiologia, Pelotas, Rio Grande do Sul, Brazil

Introduction International and Brazilian Health Agencies point to healthy eating as an important aspect of life quality and several studies have shown that a diet rich in fat increases the risk of cardiovascular diseases. In this context, we aimed to describe the prevalence of the habit of removing visible fat from meats before consumption according to socio-demographic characteristics in adult and elderly.

Methods Cross-sectional population-based study with 2706 individuals aged 20 years or more living in the urban area of Pelotas, Rio Grande do Sul, Brazil. The habit of removing visible fat from meats before eating it, defined as trimming apparent fat red meats and poultry skin, was assessed through interview reporting meat consumption considering a 12 months recall period. The sociodemographic variables were gender, skin colour, age, education and family income in tertiles.

Results Approximately $58 \%$ of interviewees were women, $81 \%$ Caucasians and about $50 \%$ reported nine or more years of study. The prevalence of the habit of removing visible fat from meats before consumption was $48 \%$ (95\% CI: 45.2 to $50.2 \%$ ), higher among women $(71 \%)$. This habit was more frequent among the Caucasians respondents (83\%), aged 60 years or older $(29 \%)$, who studied for nine to eleven years $(30 \%)$ and belonging to the highest tertile income (37\%).

Conclusion The men and individuals with lower family income and education presented the smaller prevalence of the habit of removing visible fat from meats before consumption. These results can guide public policy health for promoting strategies in this group.

\section{P2-271 SOCIOECONOMIC DEVELOPMENT AND SEX DIFFERENCES IN ISCHAEMIC HEART DISEASE MORTALITY. AN AGE- PERIOD-COHORT ANALYSIS OF THE HONG KONG CHINESE}

doi:10.1136/jech.2011.142976k.4

R Chung, C M Schooling, ${ }^{*}$ B Cowling, G Leung. The University of Hong Kong, Hong Kong, Hong Kong

Introduction An epidemic of premature ischaemic heart disease (IHD) in men usually emerges with economic development. The 